\title{
Comparison of Ferritin Measurement Performance Through Immunoturbidimetric and Chemiluminescence Methods in Patients with Critical Ferritin Levels
}

\author{
Nermin Dindar Badem* \\ Department of Medical Biochemistry, Faculty of Medicine, Kırıkkale University, Kırıkkale, Turkey \\ *Corresponding Author: Nermin Dindar Badem, Department of Medical Biochemistry, Faculty of Medicine, Kırıkkale University, Kırıkkale, \\ Turkey.
}

Received: June 27, 2019; Published: July 26, 2019

DOI: $10.31080 /$ ASMS.2019.03.0364

\begin{abstract}
The most widely used method of serum ferritin measurement, particle-enhanced turbidimetric immunoassay (PETIA) was compared with the selected reference method, electrochemiluminiscence immunassay (ECLIA), to assess accuracy, precision, reliability, and effectiveness in the cases of iron deficiency and iron overload. Serum ferritin levels of 261 patients were measured using both methods. Within-run imprecisions and inter-assay precisions of the two methods were analyzed by testing the control samples at low and high concentrations and were given as CV\%. Patients were split into three subgroups as iron deficiency ( $<15 \mu \mathrm{g} / \mathrm{L})$, normal $(15-$ $150 \mu \mathrm{g} / \mathrm{L}$ ) and iron overload (>150 $\mu \mathrm{g} / \mathrm{L}$ ), based on WHO's cut-off values. Carryover effect in Cobas C501 using the PETIA method and Cobas E601 using the ECLIA method was also investigated. Although a strong positive correlation was identified between these methods ( $r=0.998 ; p<0.0001)$, there was also a statistically significant difference $(p<0.001)$. The correlation of the ferritin results were better for the normal iron load and iron overload groups compared to the iron deficiency group, while the differences were found to be statistically significant for the iron deficiency and iron overload groups. The bias was demonstrated using the BlandAltman plot analysis. For both instruments, carryover was found to be negligible as the differences were statistically insignificant ( $>0.05$ ) and CO (\%) values were less than 1\%. For patients with critical ferritin concentrations (iron deficiency anemia, siderosis and malignancy), ECLIA method is suggested, as PETIA method's results were lower than actual and ECLIA had better precision and a wider measurement interval.
\end{abstract}

Keywords: Ferritin, Iron Deficiency; Electrochemiluminiscence Immunoassay; Particle Enhanced Turbidimetric Immunoassay; Carryover

\section{Introduction}

Ferritin is the primary iron storage protein in the human body [1]. It can be found mainly in the liver, spleen and bone marrow and used in iron recycling for hematopoiesis [1,2]. It is a molecule consisting of 24 apoferritin monomers forming a hollow spherical shape and weighing about $450 \mathrm{kDa}$ [3]. When compared to transferrin which is capable of containing two iron atoms, a single ferritin molecule can carry 4000 iron atoms, potentially making ferritin a much more effective iron transfer system [3,4]. Studies have shown that extracellular ferritin could function as an iron conveyer to cells [4].
As the cellular iron storage protein, ferritin can also be found in either the serum or the plasma [5-7]. It can be measured in the serum, plasma and also in erythrocyte [7]. It is accepted that ferritin measurement is the most reliable way of assessing the iron storage [5-7]. Low ferritin levels indicate iron deficiency and its measurements are used in the diagnosis, prognosis and treatment of iron deficiency anemia, while high ferritin levels indicate excessive iron and are valuable in diagnosis of siderosis [5-8]. Serum ferritin is also an acute phase reactant, which increases during acute and chronic inflammation [7]. Excess iron is stored in hepatocytes 
Comparison of Ferritin Measurement Performance Through Immunoturbidimetric and Chemiluminescence Methods in Patients with Critical Ferritin Levels

through ferritin [8]. Noticeable increases in ferritin levels could also indicate cellular ferritin release with hepatic damage [7].

Several methods have been reported for the measurement of serum ferritin. These include immunoradiometric assay (IRMA) [9], radioimmunoassay (RIA) [10], enzyme-linked immunosorbent assay (ELISA) [11], fluorescence polarization immunoassay [12], microparticle enzymoimmunoassay (MEIA), electrochemiluminiscence immunassay (ECLIA) [13], quantum dots [14], plasma-mass spectrometry [15] and micro array based technologies [15].

ECLIA can provide the accuracy and specificity required to detect ferritin of several $\mu \mathrm{g} / \mathrm{L}$ in patients with smaller iron deposits [5]. However, it may also be time consuming and require specialized equipment [5]. Turbidimetric immunoassay [16] is another method developed to rapidly measure analytes of higher concentrations at $\mathrm{mg} / \mathrm{L}$ levels [5]. Concentration ranges for turbidimetric analyses could be expanded [5] by making use of the light scattering property of the immunoaggregates formed by the antibodies attaching to latex particles as in enhanced turbidimetric immunoassay (PETIA) [17] or latex agglutination immunoassay [18]. These methods do not require specific instruments besides an automatic analyzer, and have advantages like reduced time requirements due to integration with other clinical chemistry analyses and lower costs [5]. Besides serum ferritin, serum iron, total iron-binding capacity, soluble transferrin receptor (sTfR), zinc, protoporphyrin, hepcidin and transferrin saturation (TSAT) are other indicators of iron condition. These analytes, along with serum ferritin, are also proposed to be reliably measured by turbidimetric immunoassay, with the same lower cost and time requirement advantages $[5,19,20]$.

The purpose of this work is to compare two of the most widely used measurement methods of serum ferritin concentrations, PETIA method used by Cobas C501 autoanalyzer and the selected reference method, ECLIA, used by Cobas E601 analyzer, in terms of their precisions, accuracies and reliability and assess their effectiveness in diagnosis and prognosis of iron deficiency anemia and iron overload.

\section{Materials and methods}

This work was planned as a prospective study in the Department of Medical Biochemistry, Kirıkkale University and was approved by the ethics board (Reference Number: 2018-15/11). For the patients, criteria for inclusion were being over the age of 18 and having currently measured ferritin levels. Exclusion criteria consisted in having ferritin levels outside the measurement interval and having high levels of CRP.

\section{Characteristics of the analyzers}

Particle-Enhanced Turbidimetric Immunoassay (PETIA) (Cobas C501 Roche Diagnostic Tina-quant Ferritin Gen.4) [21]: The automatic Roche ferritin test relies on the principle of immunological agglutination through enhancing the reaction using latex. The antiferritin reactive consists of a water-based matrix that includes latex particles covered with anti-human ferritin antibodies sourced from rabbits. Ferritin molecules in the sample combine with the latex particles covered with anti-ferritin antibodies. The precipitate can be analyzed turbidimetrically at $570 / 800 \mathrm{~nm}$. The analysis duration is 10 minutes. The polyclonal antibodies used in this test are specific to ferritin sourced from human liver, but they are also able to recognize ferritin from the human spleen. The antibodies do not show cross reactivity with human ferritin $\mathrm{H}$ subspecies. Roche/ Hitachi Cobas C 501 analyzer, which employs the PETIA method, lists the reference intervals for females at $15-150 \mu \mathrm{g} / \mathrm{L}$ and males at $30-400 \mu \mathrm{g} / \mathrm{L}$, while the measurement interval is $5-1000 \mu \mathrm{g} / \mathrm{L}$ [22]. This method is standardized according to the National Institute for Biological Standards and Control (NIBSC, WHO) Elecsys Ferritin test (immunological method).

Electrochemiluminescence Immunoassay (ECLIA) (Cobas E601 Roche Diagnostic): Serum ferritin forms a sandwich complex between the mouse biotinized monoclonal anti-ferritin antibody and the ruthenium marked mouse monoclonal anti-ferritin antibody. After the addition of streptavidin covered microparticles, the complex is bound to the solid phase through the interaction between biotin and streptavidin. The reaction mixture is then aspirated into the measurement cell where the microparticles attach to the electrode surface magnetically. Afterwards, the non-bound materials are washed away. Applying voltage to the electrode results in chemiluminescence, which is then measured using a photon counter (photomultiplier). The analysis duration is 18 minutes. Cobas E601 analyzer, which employs the ECLIA method, lists the reference intervals for females at 13-150 $\mu \mathrm{g} / \mathrm{L}$ and males at 30-400 $\mu \mathrm{g} / \mathrm{L}$, while the measurement interval is $0.5-2000 \mu \mathrm{g} / \mathrm{L}$ [22]. This method is standardized according to NIBSC 80/602 "Reagent for Ferritin (human liver)". 
Cobas C501 employing the PETIA method and Cobas E601 employing the ECLIA method are instruments of the Roche Diagnostic Cobas $^{\circledR} 6000$ analyzer series. Cobas C501 is a fully automated biochemistry instrument capable of photometric and immunoturbidimetric measurements, it also includes a contact-free ultrasonic mixing system that eliminates the likelihood of carryover. Cobas E601 is an immunochemistry analyzer using the ECL technology. It eliminates carryover by using (carryover free) disposable tips and containers.

Study design

The comparison study was conducted according to the Clinical and Laboratory Standards Institute (CLSI) protocol EP09-A3 [23]. Serum ferritin levels of 261 patients (184 female,77 male) of ages between 18 and 75 were measured with two different methods using the same samples. All the samples were collected into plastic serum tubes containing VACUETTE ${ }^{\circledR}$ gel with a clot activator. Serums were separated at room temperature by being subjected to 10 minutes of centrifugation at $1300 \mathrm{~g}$ and were analyzed afterwards within two hours using Roche/Hitachi Cobas C501 (Roche Diagnostics, Mannheim, Germany) and Cobas E601 (Roche Diagnostics, Mannheim, Germany).

Accuracy and precision

To assess the accuracy and compare the results of ferritin measurements from Cobas C501 (PETIA) and Cobas C601 (ECLIA), both instruments were calibrated with calibrators acquired from the manufacturer and all 261 samples were analyzed in duplicates in each instrument.

As defined in EP15-A3 protocol from the Clinical and Laboratory Standard Institute guideline [23], within-run imprecision and inter-assay precision of the methods were conducted by testing control samples at low and high concentration levels and were expressed as $\mathrm{CV} \%$.

The total error is comprised of two factors: systematic error (bias) and random error (imprecision). Forming an appropriate error budget allows the control of accuracy and precision in the laboratory results. It was assumed that $50 \%$ of the total acceptable error is by systematic error (bias) and $25 \%$ is by random error. Bias between the two methods was compared to total acceptable error limits listed by American Association of Bioanalysts (AAB) $(4.5 \mu \mathrm{g} / \mathrm{L}$ or $30 \%)$ and Royal College of Pathologists of Australasia (RCPA) ( \pm 4.0 up to $27 \mu \mathrm{g} / \mathrm{L} ; 15 \%>27 \mu \mathrm{g} / \mathrm{L})$.

\section{Linearity and carryover}

Linearity of the measurement methods were evaluated separately for each instrument. Samples of high and low ferritin levels were mixed at different ratios to generate a series of samples within the concentration interval. Serum pools of low $(65.50 \mu \mathrm{g} / \mathrm{L}$ for Cobas C501 and 72.23 $\mu \mathrm{g} / \mathrm{L}$ for Cobas E601) and high (677.70 $\mu \mathrm{g} / \mathrm{L}$ for Cobas C501 and 858.2 $\mu \mathrm{g} / \mathrm{L}$ for Cobas E601) ferritin concentrations were prepared. Ferritin concentration in the low concentration pool was below the clinical decision threshold (Xcl) of $150 \mu \mathrm{g} / \mathrm{L}$. The allowable bias $\left(\mathrm{B}_{\mathrm{A}}\right)$ at this threshold is 0.075 [24].

To examine the potential effect of specimen to specimen carryover, two different sequences were used. For each sequence, samples of high $(\mathrm{H})$ and low $(\mathrm{L})$ ferritin concentrations were placed one after the other and measured twice for each of the instruments. First sequence was L1 L2 L3 H1 H2 L4 H3 H4 L5 L6 L7 L8 H5 H6 L9 H7 H8 L10 H9 H10 L11 and the second one was H1 H2 H3 H4 L1 L2 L3 L4. For the first sequence, Student $t$ test was used to determine the statistical significance of carryover between the two groups, Ls following Ls (L/L) and Ls following Hs (H/L). The second sequence was used to calculate $\mathrm{CO} \%$.

Subgroup analysis

The collected samples were separated into three subgroups following the WHO cut-off values established based on the ECLIA method [7] and were assessed accordingly. The three subgroups are as follows:

Group 1 (ID): Iron deficiency group (to evaluate the diagnostic accuracy of ferritin in determining iron deficiency at the current WHO cut-off value for females, $<15 \mu \mathrm{g} / \mathrm{L}$ )

Group 2 (IN): Normal iron load group (ferritin levels between $15-150 \mu \mathrm{g} / \mathrm{L})$

Group 3 (IO): Iron overload group (to evaluate the diagnostic accuracy of ferritin in determining iron overload at current WHO cut-off value for females, $>150 \mu \mathrm{g} / \mathrm{L}$ )

The first group consisted of 89 patients with iron deficiency anemia, the second group consisted of 115 patients with normal ferritin levels and the third group consisted of 57 patients with advanced siderosis, acute/chronic inflammation or malignity. 
Comparison of Ferritin Measurement Performance Through Immunoturbidimetric and Chemiluminescence Methods in Patients with Critical Ferritin Levels

\section{Statistical analyses}

Statistical analyses were conducted using the MedCalc software (Mariakerke, Belgium). The significance of the difference between the methods were analyzed using the Paired-Samples T test. The relationship between the methods was assessed using Pearson correlation and Passing-Bablok Regression Analysis. For the differences between the measurement results, Bland and Altman analysis was conducted. On the Bland-Altman graph, $\mathrm{x}$-axis showed the tween the two methods.

\section{Results}

Accuracy and precision

The analyte concentrations were within the measurement intervals. The manufacturer's stated variation coefficients were confirmed in the laboratory through within-run imprecision and inter-assay precision studies (Table 1).

\begin{tabular}{|l|c|c|c|c|c|}
\hline & & \multicolumn{2}{|c|}{ Cobas C501 } & \multicolumn{2}{c|}{ Cobas E601 } \\
\hline & & Laboratory & Manufacturer & Laboratory & Manufacturer \\
\hline CV\%r & Level 1 & 1.6 & 0.8 & 3.0 & 1.7 \\
\hline & Level 2 & 2.2 & 0.6 & 3.6 & 1.6 \\
\hline CV\%1 & Level 1 & 1.7 & 1.1 & 1.5 & 2.5 \\
\hline \multicolumn{7}{|l}{} & Level 2 & 2.4 & 1.3 & 3.4 & 2.8 \\
\hline CV\%r, within-run imprecision; CV\%l, inter-assay precision coefficient of variation \\
\hline
\end{tabular}

Table 1: Assessment of method precision.

Method comparison results are given in Table 2. Patients' results in means and standard deviations for each instrument were as follows; $141.55 \pm 285.68 \mu \mathrm{g} / \mathrm{L}$ for Cobas E601 and $120.94 \pm$ $243.92 \mu \mathrm{g} / \mathrm{L}$ for Cobas C501. While a strong positive correlation was identified between Cobas E601 and Cobas C501 results ( $\mathrm{r}=$ 0.998), two methods also showed a statistically significant difference $(\mathrm{p}<0.0001)$. The regression equation was calculated as $\mathrm{y}=$ $0.311+0.852 x\left(r^{2}=0.996\right)$. The calculated intercept of 0.311 (ide- ally 0 ) and slope of 0.852 (ideally 1) shows the linear correlation and consistence between the two methods. Passing-Bablok regression graph and analysis are given in Figure 1 and Table 2. In the Bland-Altman graph, as ferritin concentrations increase, the difference between the two methods becomes proportionally smaller. Mean and the standard deviation of the differences were $-15.2 \pm$ $21.2 \mu \mathrm{g} / \mathrm{L}$ (95\% CI 6.0 / -36.4) (Figure 1).

\begin{tabular}{|l|l|l|}
\hline \multicolumn{2}{|l|}{ Cobas E601 } & Cobas C501 \\
\hline Mean \pm St. dev. $(\mu \mathrm{g} / \mathrm{L})$ & $141.55 \pm 285.68$ & $120.94 \pm 243.92$ \\
\hline Median, $(\mu \mathrm{g} / \mathrm{L})$ & 32.41 & 29.60 \\
\hline Min-Max ( $\mu \mathrm{g} / \mathrm{L})$ & $1.77-1989.00$ & $1.20-1657.70$ \\
\hline Paired samples t-test & \multicolumn{2}{|l|}{} \\
\hline Mean difference (95\%CI) & $-20.60(-15.15$ to -26.06$)$ & \\
\hline p value & $<0.0001$ & \\
\hline Correlation Analysis & \multicolumn{2}{|l}{} \\
\hline Pearson's correlation (r), (95\%CI) & 0.998 & $\mathrm{p}<0.0001$ \\
\hline Regression Equation & $\mathrm{r}^{2}: 0.996, \mathrm{y}=0.311+0.852 \mathrm{x}$ \\
\hline Slope, (95\%CI) & $0.852(0.846$ to 0.859$) \quad \mathrm{p}=0.764$ \\
\hline Intercept, (95\%CI) & $0.311(-1.723$ to 2.344$)$ \\
\hline Passing-Bablok Regression Analysis & $\mathrm{y}=-0.1476+0.8695 \mathrm{x}$ \\
\hline Regression Equation & $0.8695(0.8590$ to 0.8830$)$ \\
\hline Slope, (95\%CI) & $-0.1476(-0.4312$ to 0.0444$)$ \\
\hline Intercept, (95\%CI) &
\end{tabular}

Table 2: Statistical analyses of method comparison. 


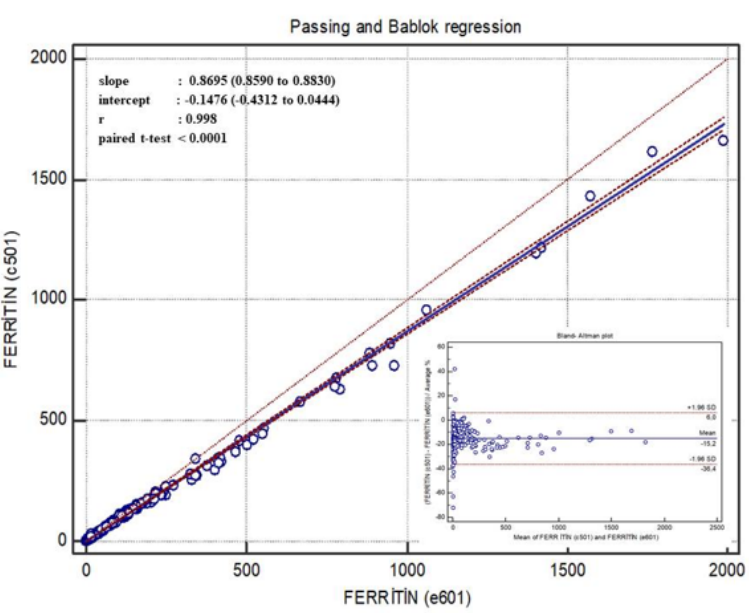

Figure 1: Passing-Bablok regression graph of Cobas C501 and Cobas E601 ferritin results, including the Bland-Altman graph for the differences (straight line is the mean of differences, dashed lines show the $95 \%$ confidence interval)

\section{Linearity and carryover}

Results of the first sequence (high and low concentrations) part showed that, for ferritin, the average of the differences between $\mathrm{H} / \mathrm{L}$ and L/L groups were significantly below the $\mathrm{B}_{\mathrm{A}}$ level. Additionally, the t test showed that the two groups were not statistically significantly different $(\mathrm{p}>0.05)$. CO (\%) values calculated from the second sequence were found to be less than $1 \%$. In light of these factors, for both sequences while using both instruments, carryover levels were found to be negligible for ferritin. Results of the CO (\%) calculations for the first and second sequences are shown on Table 3.

\begin{tabular}{|l|c|c|}
\hline & $\begin{array}{c}\text { C501 } \\
\text { (FERRITIN) }\end{array}$ & $\begin{array}{c}\text { E601 } \\
\text { (FERRITIN) }\end{array}$ \\
\hline L/L $(n=10)$ & $67.47 \pm 0.61653$ & $73.186 \pm$ \\
& & 1.36682 \\
\hline H/L $(n=10)$ & $67.09 \pm 0.77093$ & $72.27 \pm$ \\
& & 1.07527 \\
\hline Difference between Means & 0.38 & 0.916 \\
\hline Allowable Bias $\left(\mathrm{B}_{\mathrm{A}}\right)$ & 0,4005 & 0.60467 \\
\hline Statistical Significance $(\mathrm{p})$ & 0.367 & 0.164 \\
\hline
\end{tabular}

\begin{tabular}{|l|c|c|}
\hline & $\begin{array}{c}\text { C501 } \\
\text { (FERRITIN) }\end{array}$ & $\begin{array}{c}\text { E601 } \\
\text { (FERRITIN) }\end{array}$ \\
\hline Carryover (\%CO) & 0.03116 & 0.05445 \\
\hline
\end{tabular}

Table 3: Ferritin carryover results for the first and second sequences.

\section{Subgroup analysis}

Method comparison results of the subgroups are given in Table 4. Correlation between the two instruments was higher for the IN (group 2) and IO (group 3) groups, compared to the ID group (group 1). For ID and IO groups, the difference was statistically significant and the Bland-Altman plot analysis showed the bias between the results. The mean and the standard deviation of the differences were $-20.1 \pm 37.8 \mu \mathrm{g} / \mathrm{L}$ (95\% CI $17.8 /-57.9)$ for the ID group, $-11.0 \pm 15.6 \mu \mathrm{g} / \mathrm{L}(95 \% \mathrm{CI} 4.7 /-26.6$ ) for the IN group and $-17.3 \pm 11.8 \mu \mathrm{g} / \mathrm{L}(95 \% \mathrm{CI}-5.5 /-29.1)$ for the IO group (Table 4 , Figure 2).
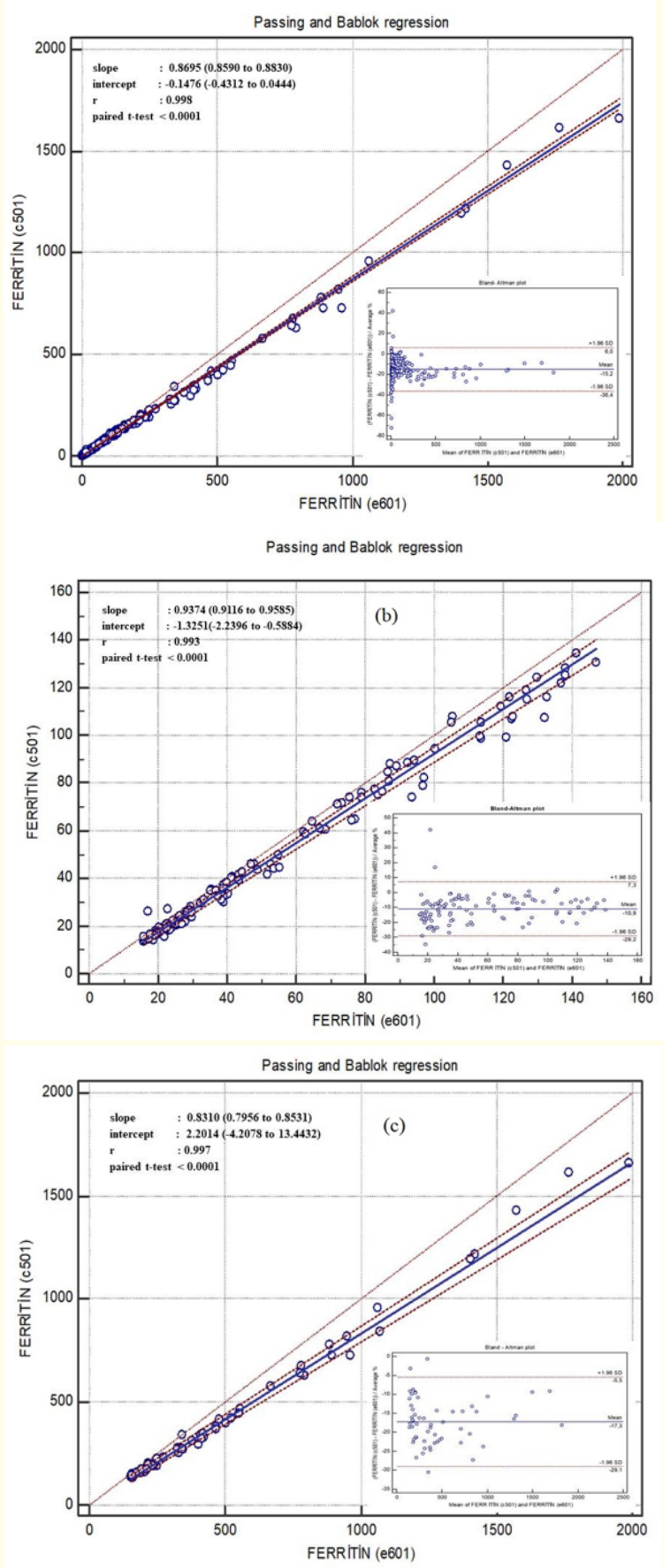

Figure 2: Passing-Bablok regression graph of Cobas C501 and Cobas E601 for the results of the three concentration groups including the Bland-Altman graph for the differences (straight line is the mean of differences, dashed lines show the $95 \%$ confidence interval) (a: iron deficiency group, b: normal iron load group, c: iron overload group). 
Comparison of Ferritin Measurement Performance Through Immunoturbidimetric and Chemiluminescence Methods in Patients with Critical Ferritin Levels

165

\begin{tabular}{|c|c|c|c|c|c|c|}
\hline \multirow[t]{2}{*}{ Groups } & \multicolumn{2}{|c|}{$\begin{array}{l}\text { Iron Deficiency }(<15 \mu \mathrm{g} / \mathrm{L}) \\
(\mathrm{n}=89)\end{array}$} & \multicolumn{2}{|c|}{$\begin{array}{l}\text { Normal Iron Load }(20-150 \mu \mathrm{g} / \mathrm{L}) \\
(\mathrm{n}=115)\end{array}$} & \multicolumn{2}{|c|}{$\begin{array}{l}\text { Iron Overload }(>150 \mu \mathrm{g} / \mathrm{L}) \\
(\mathrm{n}=57)\end{array}$} \\
\hline & Cobas E601 & Cobas C501 & Cobas E601 & Cobas C501 & Cobas E601 & Cobas C501 \\
\hline Mean \pm St.dev. $(\mu \mathrm{g} / \mathrm{L})$ & $8.39 \pm 2.95$ & $7.01 \pm 2.69$ & $58.24 \pm 38.69$ & $52.77 \pm 35.57$ & $517.56 \pm 435.34$ & $436.38 \pm 377.09$ \\
\hline Median, $(\mu \mathrm{g} / \mathrm{L})$ & 8.33 & 6.90 & 41.43 & 39.20 & 346.30 & 296.00 \\
\hline \multirow[t]{3}{*}{$\operatorname{Min}-\operatorname{Max}(\mu \mathrm{g} / \mathrm{L})$} & $1.77-14.16$ & $1.20-12.60$ & $15.84-146.90$ & $13.60-134.30$ & $151.60-1989.00$ & $130.80-1657.70$ \\
\hline & \multicolumn{2}{|c|}{ Paired Samples T-Test } & \multicolumn{2}{|c|}{ Paired Samples T-Test } & \multicolumn{2}{|c|}{ Paired Samples T-Test } \\
\hline & \multicolumn{2}{|c|}{$\begin{array}{l}\text { Mean Difference (95\%CI) } \\
-1,3835(-1.2058 \text { to }-1.5612)\end{array}$} & \multicolumn{2}{|c|}{$\begin{array}{l}\text { Mean Difference (95\%CI) } \\
-5.4640(-4.4854 \text { to- } 6.4426)\end{array}$} & \multicolumn{2}{|c|}{$\begin{array}{l}\text { Mean Difference (95\%CI) } \\
-81.1754 \text { (-63.4614 to -98.8895) }\end{array}$} \\
\hline $\mathrm{p}$ value & \multicolumn{2}{|l|}{$<0.0001$} & \multicolumn{2}{|l|}{$<0.0001$} & \multicolumn{2}{|l|}{$<0.0001$} \\
\hline \multicolumn{7}{|l|}{ Correlation Analysis } \\
\hline $\begin{array}{l}\text { Pearson's correlation } \\
\text { (r) }\end{array}$ & \multicolumn{2}{|l|}{0.959} & \multicolumn{2}{|l|}{0.993} & \multicolumn{2}{|l|}{0.997} \\
\hline Regression Equation & \multicolumn{2}{|c|}{$r^{2}: 0.920, y=-0.337+0.875 x$} & \multicolumn{2}{|c|}{$\mathrm{r}^{2}: 0.987, y=-0.421+0.913 x$} & \multicolumn{2}{|c|}{$r^{2}: 0.993, y=-10.474+0.863 x$} \\
\hline Slope, $(95 \% \mathrm{CI})$ & \multicolumn{2}{|c|}{0.875 (0.820 to 0.930$) \quad \mathrm{p}<0.0001$} & \multicolumn{2}{|c|}{$0.913(0.894$ to 0.933$) \quad \mathrm{p}<0.0001$} & \multicolumn{2}{|c|}{$0.863(0.845$ to 0.882$) \quad \mathrm{p}<0.0001$} \\
\hline Intercept, $(95 \% \mathrm{CI})$ & \multicolumn{2}{|c|}{$-0.337(-0.825$ to 0.151$) \mathrm{P}=0.174$} & \multicolumn{2}{|c|}{$-0.421(-1.801$ to 0.960$) \quad \mathrm{P}=0.547$} & \multicolumn{2}{|c|}{$\begin{array}{l}-10.474(-23.163 \text { to } 2.214) \\
P=0.104\end{array}$} \\
\hline \multicolumn{7}{|c|}{ Passing-Bablok Regression Analysis } \\
\hline Regression Equation & \multicolumn{2}{|c|}{$y=-0.6147+0.9073 x$} & \multicolumn{2}{|c|}{$y=-1.3251+0.9374 x$} & \multicolumn{2}{|c|}{$y=2.2014+0.8310 x$} \\
\hline Slope, $(95 \% \mathrm{CI})$ & \multicolumn{2}{|c|}{$0.9073(0.8529$ to 0.9607$)$} & \multicolumn{2}{|c|}{$0.9374(0.9116$ to 0.9585$)$} & \multicolumn{2}{|c|}{$0.8310(0.7956$ to 0.8531$)$} \\
\hline Intercept, $(95 \% \mathrm{CI})$ & \multicolumn{2}{|c|}{$-0.6147(-1.0148$ to -0.1782$)$} & \multicolumn{2}{|c|}{$-1.3251(-2.2396$ to -0.5884$)$} & \multicolumn{2}{|c|}{$2.2014(-4.2078$ to 13.4432$)$} \\
\hline
\end{tabular}

Table 4: Statistical comparison of the concentration groups.

\section{Discussion}

This study showed that while both methods are viable for patients with normal ferritin levels, for patients with iron deficiency anemia, siderosis and malignity whose ferritin levels are either below or above normal, ECLIA method would be more appropriate during diagnosis and prognosis. PETIA method was found to measure the ferritin levels lower than the actual case, especially more prevalent in the ID and IO groups. Additionally, ECLIA method proved to have higher precision while also being able to measure a wider range of concentrations.

Measurement of specific proteins has become an important part of routine clinical chemistry laboratories. As a valuable way of assessing iron deposits in the body, demand for serum ferritin measurements has significantly increased. Serum ferritin is an early indicator of the iron deposits and combined with other tests evaluating the iron condition, it is the most specific indicator for the depleted iron deposits [20].
Garcia-Casal., et al. in their comprehensive review and metaanalysis study on the performances and comparability of laboratory assays for the determination of human serum or plasma ferritin concentrations, have reported that a golden standard for ferritin measurements has not been defined yet, thus, ferritin methods cannot be compared to a reference method; instead, individual method performances and comparisons between current methods that include consistent data were assessed [26]. They have stated that because of limited data and quality, meta-analysis of important statistical indicators such as repeatability, high dose hook effect, carryover and Bland-Altman statistics was not possible [26]. They have also identified that more than $98 \%$ of the included studies did not employ appropriate method comparison techniques like Passing-Bablok regression, Spearman correlation or concordance methods [26]. It was stated that these limitations were caused by the studies focusing on the similarities between the methods instead of confirming their accuracies [26]. In terms of patient care, public health research and assessment of the impact of a treatment, 
it was advised that once a method was selected, it would be better to continue with it [26]. In this method (and corresponding instrument) comparison study, statistical methods like Bland-Altman plot (mean differences and CV), repeatability, carryover and Passing-Bablok regression could be employed. It was determined that there were statistically significant differences between the methods and agree that a single method should be used during diagnosis, prognosis and throughout patient care.

Molinario., et al. in their recent study, have compared a new latex immunoturbidimetric ferritin assay conducted using BA 400 LED Technology (BioSystems S.A.) with a chemiluminescent microparticle immunoassay conducted on Architect i4000 (Abbott Diagnostics) [20]. They have reported that the latex immunoturbidimetric ferritin assay could be conducted using BA 400 LED Technology, or other clinical chemistry instruments, this way, measurements such as serum iron, total iron binding capacity, soluble transferrin receptor and transferrin saturation index could be done simultaneously alongside the serum ferritin measurement, using a single sample on the same device [20]. In a study on analyses using the latex agglutination method in clinical chemistry analyzers, Kotajima., et al. found that the ferritin results were correlated to those from the enzymoimmunoassay (EIA) method [27]. They have stated that accurate ferritin results were obtained on a broad range, thus, this method could be used in diagnosing iron deficiency anemia, siderosis and malignancy [27]. They have also claimed that by using the latex agglutination method on clinical chemistry analyzers, ferritin could be measured simultaneously with the other parameters of iron deficiency anemia and clinicians could obtain the results faster [27]. For this study, although a strong correlation was observed between the PETIA and ECLIA, during the measurement of the standards' concentrations, PETIA was found to measure the ferritin levels lower than actual. This may lead to misleading results, especially for iron deficiency and iron overload cases.

Zhang., et al. compared serum ferritin measurements in two analyzers using the immunoassay method (Architect i2000 and Cobas E601), they have found that although a strong correlation ( $\mathrm{r}$ $=0.99$ ) was observed between the results of the two accurate and analytically well-performing analyzers, they were different [28]. With this work, statistically significantly different results from correlated methods were also observed, especially for the ID and IO groups. This is another reason why following the same measurement method throughout a case is suggested.
In the assessments during the sub-group analysis, it was found that 5 patients had iron deficiency according to the PETIA method, while they had normal iron levels according to the ECLIA method. Similarly, 8 patients had normal iron levels when measured by the PETIA method, however, ECLIA method showed them to have iron overload instead. Overall, 13 patients out of the studied 261 were classified differently ( $\sim 5 \%)$. It is believe that this discrepancy could lead to misdiagnosis.

For ferritin measurements, contrary to other researchers, a significant difference in measurement times was not experienced. Currently, owing to the online preanalytical systems, it is also possible to simultaneously measure all the iron parameters from the same sample by linking clinical biochemistry instruments together. The matter of importance is the accurate and reliable care of patients. Thus, for ferritin measurements, PETIA, a method of lower precision, could be used for scanning purposes. However, during the care of patients with iron deficiency anemia, siderosis and malignancy, ECLIA, which showed better precision and reliability, should be preferred.

\section{Conclusion}

It was observed that serum ferritin levels measured by the immunoturbidimetric method are strongly correlated with the results from the ECLIA method. However, the ECLIA method for patient groups with either low or high ferritin levels (iron deficiency anemia, siderosis, and malignity) is recommended as it was demonstrated to have a better accuracy, precision and a wider measurement interval. For consistency and mitigation of errors, a single method (and instrument) needs to be used throughout the duration of a given patient's case.

\section{Limitations}

In this study, within-run imprecision and inter-assay precision have been used as intrinsic indicators for the assessment of different ferritin measurement methods. One of the limitations was that I did not perform a recovery study with an internationally valid standard. In addition, a separate reference value study needs to be performed for each method.

\section{Acknowledgements}

The author would like to thank Zeynep Çelik, Seydiali Peker and Dr. Uğur Tiftikçi for their assistance and guidance in this research. 


\section{Declaration of Competing Interests}

The author declared no potential conflicts of interest with respect to the research, authorship, and/or publication of this article.

\section{Funding}

This research received no specific grant from any funding agency in the public, commercial, or not-for-profit sectors.

\section{Bibliography}

1. Jacobs A and Worwood M. "Ferritin in serum. Clinical and biochemical implications". The New England Journal of Medicine 292.18 (1975): 951-956.

2. Forman DT and Parker SL. "The Measurement and Interpretation of Serum Ferritin". Annals of Clinical and Laboratory Science 10.4 (1980): 345-350.

3. Ashley E. "Ferritin (serum, plasma)". Association for Clinical biochemistry (2012): 1-8.

4. Wang W., et al. "Serum ferritin: past, present and future". Biochimica et Biophysica Acta 1800.8 (2010): 760-769.

5. Gomez F., et al. "Evaluation of a Particle-Enhanced Turbidimetric Immunoassay for the Measurement of Ferritin: Application to Patients Participating in an Autologous Blood Transfusion Program". Clinical Biochemistry 33.3 (2000): 191-196.

6. Daru J., et al. "Serum ferritin as an indicator of iron status: what do we need to know?". The American Journal of Clinical Nutrition 106.6 (2017):1634-1639.

7. Garcia-Casal MN., et al. "Serum or plasma ferritin concentration as an index of iron deficiency and overload". Cochrane Database of Systematic Reviews (2015).

8. Coad J and Pedley K. "Iron deficiency and iron deficiency anemia in women". Scandinavian Journal of Clinical Laboratory Investigation 244 (2014): 82-89.

9. Addison GM., et al. "An immunoradiometric assay for ferritin in the serum of normal subjects and patients with iron deficiency and iron overload". Journal of Clinical Pathology 25.4 (1972): 326-329.

10. Halliday JW., et al. "Solid phase radioimmunoassay for serum ferritin". Clinica Chimica Acta 58.3 (1975): 207-214.

11. Conradie JD and Mbhele BEL. "Quantitation of Serum Ferritin by Enzyme-Linked. Iinmunosorbent Assay (ELISA)". SA Medical Journal 57.8 (1980): 282-287.
12. Lovgren T., et al. "Determination of hormones by time-resolved fluoroimmunoassay”. Talanta 31.10 (1984): 909-916.

13. Steitz WR. "Immunoassay labels in chemiluminescence and bioluminescence". Clinical Biochemistry 17.2 (1984): 120-124.

14. Liu P., et al. "Ultrasensitive detection of ferritin in human serum by Western blotting based on quantum dots-labeled avidin-biotin system". Proteomics 11.17 (2011): 3510-3517.

15. Yin JY., et al. "Study on the method of quantitative analysis of serum ferritin and soluble transferrin receptor with protein microarray technology". Biomedical and Environmental Sciences 25.4 (2012): 430-439.

16. Price CP., et al. "Light scattering immunoassay of specific proteins: a review". Annals of Clinical Biochemistry 20 (1983): 1-14.

17. Thakkar H., et al. "Development and validation of a particle-enhanced turbidimetric inhibition assay for urine albumin on the Dade acat analyser". Clinical Chemistry 43.1 (1997): 109-113.

18. Thompson JC., et al. "Kinetics and proposed mechanism of the reaction of an immunoinhibition, particle-enhanced immunoassay". Clinical Chemistry 43.12 (1997): 2384-2389.

19. İnce FD., et al. "Immunoturbidimetric and Electrochemiluminescence methods for ferritin measurement". Türk Klinik Biyokimya Derneği 14.1 (2016): 51-57.

20. Molinario R., et al. "Analytical evaluation of a new liquid immunoturbidimetric assay for the determination of ferritin in serum". Letter to the Editor Clinical Chemistry and Laboratory Medicine (2015): 1-3.

21. Dubois S., et al. "Eisenstoffwechsel-Diagnostik mit Boehringer Mannheim/Hitachi-Analysensystemen: Ferritin, Transferrin und Eisen". GIT Labor-Medizin 9 (1988): 468-471.

22. Lotz J., et al. "Reference Study for Ferritin Assays". Kurzmitteilung Clinicle Laboratory 43 (1997): 993-994.

23. Budd JR., et al. "Measurement Procedure Comparison and Bias Estimation Using Patient Samples, EP09-A3; Approved Guideline-3". edition. Wayne, PA, USA. Clinical Laboratory Standards Institute (2013).

24. Kaplan LA and Pesce AJ. "Clinical Chemistry Theory, Analysis, Correlation. 3”. edition Mosby, St. Louis (1996): 292-312. 
25. Peters T and Westgard J0. "Evaluation of methods". In: Tietz NW, 1. ed, Textbook of Clinical Chemistry, Philadelphia, WB Saunders (1986): 410-423.

26. Garcia-Casal MN., et al. "Performance and comparability of laboratory methods for measuring ferritin concentrations in human serum or plasma: A systematic review and meta-analysis". Plos One (2018): 1-24.

27. Kotajima N., et al. "Latex agglutination test for ferritin measurement”. Journal of Clinical Laboratory Analysis 17.6 (2003): 223-228.

28. Zhang X., et al. "A comparison study between two analyzers for determining serum ferritin". Clinical Laboratory 61.12 (2015):169-74.

Volume 3 Issue 8 August 2019

(C) All rights are reserved by Nermin Dindar Badem. 